\section{Hochsegmentierung, erblich konstitutionelle}

H. Baum

Institut für Laboratoriumsmedizin, Mikrobiologie und Blutdepot, Regionale Kliniken Holding RKH GmbH, Ludwigsburg, Deutschland

Englischer Begriff hereditary constitutional high segmentation of neutrophilic granulocytes (Undritz)
Definition Idiopathisch vermehrtes Auftreten von übersegmentierten neutrophilen Granulozyten im peripheren Blut.

Beschreibung Die erblich konstitutionelle Hochsegmentierung nach Undritz ist durch das vermehrte Vorkommen von übersegmentierten neutrophilen Granulozyten (mehr als 5 Kernsegmente) ohne gleichzeitigen Hinweis auf eine auslösende Ursache (z. B. perniziöse Anämie, Hungerzustände, nach Transfusion) charakterisiert. Sie ist, wie alle sogenannten „Rechtsverschiebungen“, ohne differenzialdiagnostische Bedeutung. 